\title{
A Logic of Interrogation Should Be Internalized in a Modal Logic for Knowledge
}

\author{
Rani Nelken and Chung-chieh Shan \\ Harvard University
}

\section{Introduction}

We propose a new, modal interpretation of questions. The idea of interpreting questions via modal logic goes back to Hintikka (1976) and Åqvist (1965), who interpret a question as a request for knowledge: "bring it about that I know whether ...". Such a request is composed of an imperative part and an epistemic part. Focusing on the latter, we interpret a question as the knowledge condition required in order to answer it completely. We will reduce the epistemic part of the meaning of both yes-no questions and $w h$-questions to statements of the form "it is known whether" or "it is in the common ground that ...". For instance, for a yes-no question such as Is Alice quitting?, the meaning is "it is known that Alice is quitting or it is known that Alice is not quitting".

Several different approaches have been suggested in linguistic semantics for modeling questions.

1. It is popular to follow Hamblin (1973) and Karttunen (1977) (hereafter HK) in taking a question to denote its set of partial answers, or partial true answers. For instance, for the wh-question Who's quitting?, these would be answers of the form: Alice is quitting, or Alice and Bob are quitting.

2. Groenendijk and Stokhof $(1984,1996$; hereafter GS) propose a more parsimonious approach in which the answers in the set are required to be complete and mutually exclusive-in other words, a partition of possible worlds in the space of logical possibilities. For the same question, these answers would be Nobody is quitting, Just Alice is quitting, etc.

3. In contrast to these firmly intensional question denotations, Nelken and Francez (2000, 2002; hereafter NF) propose an extensional interpretation. The meaning of the same question is $r$ ("resolved") if it is known for every person in the domain whether he or she is quitting. Otherwise, it is $u r$ ("unresolved").

In this paper, we bridge these theories and combine their advantages. We begin by presenting the basic approach in Section 2. In Section 3 we delve deeper into the denotation of questions. In particular, we address what has been the main criticism against similar approaches: how to deal with embedded questions. Our theory captures GS's prized entailment relations among questions and assertions (Section 4), while also enjoying an extensional semantics like NF's (Section 5) and NF's increased expressive power for complex questions (Section 6). 
Section 7 shows how our knowledge conditions can encode strongly-exhaustive questions, as GS's partitions can, alongside weakly- and non-exhaustive questions, as HK's sets of answers can, and as Beck and Rullmann (1999) call for. Section 8 connects our modal perspective to families of subquestions (Beck and Sharvit 2002; Sharvit and Beck 2001). Finally, Section 9 shows that games of interrogation (Groenendijk 1999) are easily expressed and extended to multi-player settings in our approach.

\section{From knowing to asking}

The basic ingredients of our proposal are found in your favorite first-order modal logic for knowledge. Take a necessity operator $\square$, which can be read as "it is known that" or "it is in the common ground that". Assertions are formulae of the form $\square \phi$. For example, for it to be asserted that Alice is quitting is for it to be in the common ground that Alice is quitting: $\square Q a$.

We impose only minimal constraints on the logic, summarized in Table 1. First, we require a normal modality to be able to reason with the logic. To use the logic as an epistemic one, we require that knowledge of a proposition implies its truth. To simplify reasoning, we further assume that the domain remains the same as in the real world, even when contemplating epistemic alternatives.

\begin{tabular}{lll}
\hline Constraint & Syntactically & Semantically \\
\hline Normal modality & $\begin{array}{l}\text { Necessitation rule } \\
\text { and K axiom }\end{array}$ & $\begin{array}{l}\text { Possible worlds } \\
\text { and accessibility }\end{array}$ \\
Knowledge must be true & $\begin{array}{l}\text { T axiom } \\
\text { Barcan formula } \\
\text { Barcan both ways }\end{array}$ & $\begin{array}{l}\text { Accessibility is reflexive } \\
\text { Constant domain }\end{array}$ \\
& and its converse & \\
\hline
\end{tabular}

Table 1: Logical constraints

For concreteness, we assume that the underlying logic is S5, which is characterized by further validating that accessibility is transitive (the 4 axiom) and symmetric (the B axiom). ${ }^{1}$

For any formula $\phi$, we write $? \phi$ as shorthand for $\square \phi \vee \square \neg \phi$. Formulae of this form encode yes-no questions. For example, to the question Is Alice quitting? we assign the semantics ?Qa, or $\square Q a \vee \square \neg Q a$. The intuition behind this assignment is that to know whether Alice is quitting is to either know that she is or know that she is not. Thus, we directly encode the meaning of the question as its knowledge condition-what it takes to know a complete answer to the question. The intensional semantics of such formulae is the standard Kripke semantics. The meaning of $\square Q a \vee \square \neg Q a$ is that all the possible worlds seen from the current one agree on $Q a$. In other words, $Q a$ is either uniformly true in all these worlds, or uniformly false in all of them. 
Similarly, we encode wh-questions as formulae of the form ? $\vec{x} . \phi$, which is shorthand for $\forall \vec{x}$. ? $\phi$, where $\vec{x}$ is one or more variables. For example, we take the meaning of Who is quitting? to be $\forall x . \square Q x \vee \square \neg Q x$. The intuition here is that to know who is quitting is to know for each person whether he or she is quitting. The intensional semantics is that all the worlds seen from the current world agree on the extension of $Q$-the set of people who quit must be the same in all the worlds. This approach is strongly exhaustive; we will refine this assumption in Section 7.

\section{Question denotations}

The main linguistic objection to reducing questions to knowledge conditions is that it seems to take knowledge as an integral part of the question meaning. This would seem to preclude treating embedding verbs like wonder and depend on. Moreover, it does not seem to distinguish between the speech acts of asking a question and asserting knowledge of the question's answer.

To address this objection, it is important to note that the modality $\square$ in a formula like $\square Q a \vee \square \neg Q a$ refers to the knowledge state of no particular agent or group. Neither does $\square$ quantify universally over all possible worlds in intensional logic. Rather, $\square$ is just an abstract modality. By slight abuse of notation, it is perhaps more accurate to say that Is Alice quitting? and whether Alice is quitting both denote the abstraction

$$
\text { 入口. } \square Q a \vee \square \neg Q a,
$$

in which $\square$ is bound by a lambda operator. We shall shortly explain the added $\lambda \square$ model-theoretically. We posit that questions "start their life" in semantic composition with this abstraction in place. To finalize a sentence meaning, this function must be applied to some epistemic modality, in other words, to some knowledge state. Performing a matrix question applies the abstraction to the implicit conversational common ground $\square_{\mathrm{CG}}$. Likewise, a question-embedding verb such as know or wonder applies the same abstraction to other knowledge states. Thus, if to wonder is to want to know, then wonder denotes

$$
\lambda q . \lambda x . \text { ' } x \text { wants that } q\left(\square_{x}\right) \text { ', }
$$

where $\square_{x}$ is the knowledge state of $x$ in an alternative world, and $q\left(\square_{x}\right)$ is the proposition that $x$ knows (the answer to) $q$.

What does it mean for a function to take a modality as argument? A modality is specified by its accessibility relation, so we could simply say that a question meaning takes an accessibility relation as argument. Questions would then be of type $\langle\langle s,\langle s, t\rangle\rangle,\langle s, t\rangle\rangle$ : functions from accessibility relations to propositions.

But we can simplify this type using our requirements on the accessibility relation. Because accessibility is transitive and symmetric in S5, and the truth value of a modal formula only depends on worlds at least indirectly accessible, we can simplify the first argument to questions from an accessibility relation to a set of 
accessible worlds. Questions are then of type $\langle\langle s, t\rangle,\langle s, t\rangle\rangle$, so that $q(W)(w)$ holds just in case the set of worlds $W$ agree on the complete answer to $q$ at the world $w$. In other words, $q(W)(w)$ holds if knowing that the actual world is in $W$ entails knowing the complete answer to $q$ at $w$. Because accessibility is reflexive by assumption, $q(W)(w)$ need only be defined when $W$ contains $w$.

All questions on our account are monotonic: it never hurts to know more. Formally, if $q$ is a question, then $q\left(W_{1}\right)(w)$ implies $q\left(W_{2}\right)(w)$ whenever $W_{1}$ is a superset of $W_{2}$.

\section{Entailment relations}

GS describe two entailment relations involving questions that any theoretical treatment of the semantics of questions should provide. Answerhood is the relation between a question and each of its possible answers. Interrogative entailment is a relation between questions. One question is said to entail another if knowing a complete answer to the first entails knowing a complete answer to the second. As with NF's theory of questions, both these relations (as well as ordinary indicative entailment) reduce to (modal) consequence: $\phi \vDash \psi$. (That is, if $\phi$ holds in every world, then $\psi$ holds in every world.)

Here are a few example answerhood entailments. Assuming the domain consists of the two individuals Alice $(a)$ and Bob $(b)$, we have the entailment:

$$
\text { Alice is quitting and Bob isn't quitting answers Who is quitting? }
$$

which can be written:

$$
Q a \wedge \neg Q b \vDash ? x . Q x \text {. }
$$

This entailment is easily verifiable: if the left hand side is true in all possible worlds, then all worlds agree on the extension of $Q$, and hence the right hand side is also true.

The strongly exhaustive nature of the semantics becomes apparent when we consider a negative answerhood example such as

$$
\exists x . Q x \forall ? x . Q x
$$

Clearly, the left hand side may be true in all possible worlds but using a different witness for the existential quantification in different worlds, thus falsifying the right hand side.

A question such as

\section{Is it either raining or not raining?}

is trivial, in the sense that it does not impose any extra knowledge conditions:

$$
\vDash ?(R \vee \neg R) \text {. }
$$


Interrogative entailment is also straightforward, as illustrated by the following examples.

Who is quitting? entails Who is not quitting?

which can be written:

$$
\text { ?x. } Q x \vDash ? x . \neg Q x \text {. }
$$

In fact, both questions are equivalent, as they have the same knowledge conditions. An example where entailment does not hold is:

\section{Who is quitting and moving away? does not entail Who is not quitting?}

since we may know the complete answer to the former question and yet not know the complete answer to the latter one.

$$
\text { ?x. } Q x \wedge M x \forall ? x . \neg Q x
$$

The examples above illustrate the empirical adequacy of the theory. In fact, we formally prove in forthcoming work that the entailment relations of GS are exactly preserved. This is a welcome result, since it shows that we can achieve GS's explanatory power using a much simpler semantics (in fact, an extensional one, as described in Section 5). The intuition behind the proof is as follows. On the GS approach, a question partitions logical space into equivalence classes, corresponding to the possible answers of the question. On our approach, in each possible world $w$ we assign the question the value "true" iff all the worlds accessible from $w$ (that is, all the worlds compatible with the agent's knowledge) agree on the answer. In other words, for each possible world, an agent may entertain only epistemic possibilities that are homogeneous with respect to the answer to the question. Thus, for a question to be resolved, the knowledge relation should respect the partition boundaries. This yields an exact correspondence between the GS partition interpretation of questions and our new modal interpretation.

By assigning truth values (of modal formulae) to questions, we gain the simplicity of NF's analysis: a uniform consequence relation that includes indicative entailment, answerhood, and interrogative entailment as particular instances. We also gain expressive power with which to account for more complex questions, as explained in Section 6. Another advantage of our modal perspective is that established techniques of modal logic are immediately available for reasoning about questions. In particular, we can apply inference procedures for modal logic to answerhood, extending ten Cate and Shan's (2002) question-answering algorithm for GS's partition semantics to any question meanings encoded as knowledge conditions. In fact, we can directly use Cerrito and Cialdea Mayer's (2001) proof procedure for first-order S4 logic. The procedure is sound and complete-given a question, it generates only and all answers. However, as one might expect, it does not always terminate-in some cases, there is an infinite number of possible answers, and no answer is most informative. 


\section{Extensional semantics}

Both HK and GS take a question to mean its answers. Because answers are propositions, such an approach inherently attributes the added complexity of intensionality to interrogative sentences, but not to indicative sentences. While there may be good reasons to adopt such an asymmetry, it is less clear what level of intensionality is actually necessary to interpret questions. To examine this issue, we propose to try to construct an extensional semantics of questions, and see how far one can take it. Even if intensionality turns out to be necessary in the end, we will better understand what phenomena in the interpretation of questions really overstep the extensional boundary and drive the need for intensionality.

NF propose an explicitly extensional interpretation of questions. They extend the domain of truth values from 2 values to 5 , organized in a bilattice along two dimensions: truth and resolvedness. ${ }^{2}$ Both indicative and interrogative sentences are assigned values from this 5-element set. Indicative sentences are assigned one of three truth values, $t, f$ and $u k$ (unknown). Interrogative sentences are assigned one of two resolvedness values, $r$ (resolved) and $u r$ (unresolved), depending on the truth value of the underlying indicative sentence:

$$
\begin{aligned}
? Q a & = \begin{cases}r & \text { if } Q a \in\{f, t\} \\
u r & \text { otherwise }\end{cases} \\
? x . Q x & = \begin{cases}r & \text { if } Q x \in\{f, t\} \text { for all values of } x \\
u r & \text { otherwise }\end{cases}
\end{aligned}
$$

Of the 5 values, the values $t$ and $r$ are designated. Entailment is defined to hold between two formulae if whenever the first formula is assigned a designated value, so is the second. This notion of entailment uniformly accounts for the different kinds of entailment (indicative, answerhood, and interrogative), and correctly predicts a wide range of empirical entailments. The NF approach can be seen as a particular interpretation of modal formulae over a domain of five values. The $\square$ operator is just a mapping from the indicative values to the interrogative ones, mapping $f$ and $t$ to $r$, and $u k$ to $u r$.

Unfortunately, the bilattice interpretation is not entirely empirically accurate. For instance, (6) is erroneously not predicted to be a trivial question using the bilattice approach; in other words, the entailment (7) should be valid but is not (Nelken and Francez 2002). This failure would appear to cast into doubt the empirical adequacy of interpreting questions extensionally.

Fortunately, our modal approach offers a new extensional interpretation of questions that preserves GS's entailment relations (and thus correctly validates (7)). Recall that propositional modal logic has the finite-model property: if a collection of formulae has a model, then it has a model with a finite number of possible worlds. This useful property does not hold in general for first-order modal (predicate) logic. However, we show in forthcoming work that the finite-model property does hold if we restrict ourselves to first-order modal formulae that encode questions. The main reason is that the modal depth (roughly, the maximum number of $\square$-nesting 
levels) of these formulae is bounded. In fact, because the modal depth is at most 1 (in other words, we never nest one $\square$ under another in order to encode questions), even two worlds are enough. More precisely, any entailment that we encounter while interpreting questions is satisfiable iff it is satisfiable with two worlds, and falsifiable iff it is falsifiable with two worlds.

We can use this result to provide a novel extensional interpretation which exactly preserves the entailment relations of GS. We can straightforwardly simulate a two-world structure using a regular (non-modal) first-order model, by using 4 truth values: $\{F F, T F, F T, T T\}$. In this model, each atomic formula is assigned one of these four values. The truth value of more complex formulae is computed by applying the regular truth tables of the logical operators in a point-wise fashion. In particular, ? computes the consensus between the two worlds: it maps $T T$ and $F F$ to $T T$; and $T F$ and $F T$ to $F F$. The double truth values thus simulate the two worlds. We illustrate this simulation with an example. Consider once again (7), repeated here:

$$
\vDash ?(R \vee \neg R) \text {. }
$$

Since the logical operators operate point-wise, $R \vee \neg R$ is $T T$ regardless of the truth value of $R$. Thus ? $(R \vee \neg R)$ is $T T$, so the entailment holds in this 4-value logic, as desired.

This novel extensional interpretation nevertheless has a certain intensional flavor, in that the 4 truth values simply encode two possible worlds. This flavor raises a more philosophical issue regarding what constitutes an extensional semantics. On one hand, if we forget about the possible worlds and just use a non-classical handful of truth values, would our semantics appear more extensional? On the other hand, if we had to encode more possible worlds, say 20 instead of 2, yielding an astronomical number of truth values, would the interpretation still qualify as extensional? Is intensionality a binary decision or is it a matter of degree?

\section{Internalized questions}

Because we let questions denote their knowledge conditions, which are just modal formulae, they can further combine with other formulae. In particular, our ? operator is internalized: as in NF's but not GS's analysis, it can apply anywhere in a question meaning, not just at the top level or under some top-level universal quantifiers. We can thus handle the following constructions using standard logical connectives.

Conjunction Do you have a license and who (else) has one? (?L $\wedge$ ?x. $L x)$.

Disjunction What's your social security number? Or what's your mother's maiden name? (?x. $S x \vee ? x . M x)$. Is it getting warmer? Or is it just me? Whereas conjunction is straightforward for GS, they get disjunction only by resorting to higher-order type-shifting from partitions to sets of sets of partitions, getting a highly complex object. 
Conditional If it's raining, may I borrow your umbrella? $(R \rightarrow ? U)$. These questions are unavailable for GS.

Universal quantification Who recommends each candidate? ( $\forall y . C y \rightarrow ? x . R x y)$. In previous theories, because questions are not propositions, they are difficult to quantify over. For instance, Karttunen uses double negation to get these readings. Since conditional questions are unavailable for GS, so is universal quantification over conditional questions.

Also by universally quantifying over conditional questions, we can express the contrast between de dicto and de re readings of which-questions (Groenendijk and Stokhof 1984), as in Alice just discovered which spies are quitting. The de dicto reading here $(\forall x . S x \rightarrow ? Q x)$ neither entails nor is entailed by the de re reading $(\forall x . ?(S x \wedge Q x))$.

The fact that our logic allows these constructions straightforwardly, whereas HK and GS struggle to get them, if they get them at all, raises an important methodological issue. There are well-known restrictions on what operators and quantifiers can scope over questions. For instance, one cannot negate questions, and only the universal quantifier can robustly scope over questions. Likewise, while conditional questions are clearly available, there is some debate in the literature over the empirical acceptability of disjunctions over questions. Szabolcsi (1997) claims that they are unavailable, whereas GS and NF accept them.

Clearly, a fully adequate theory of questions, be it of syntactic interrogatives or semantic information requests, should predict all and only the available constructions. HK and GS start off with a relatively complicated system that undergenerates, then apply additional operations that are usually not externally motivated, like higher-order type-shifting, to generate (some) more complex constructions. Of course, for the empirically unavailable combinations (such as negated questions) these theories can simply remain silent, but the empirically available combinations (such as conditional questions) necessitate complications. By contrast, our modal approach over-generates, so we have to impose additional restrictions in order to rule out the unacceptable constructions-not a difficult task, but stipulative still.

We find a logic like ours valuable, in which these unavailable combinations can at least be encoded alongside available ones for discussion. This allows us to reason about the unavailable combinations without descending into formal gibberish. For instance, we can ask why a particular construction cannot make a particular information request, hence providing some explanation for why the corresponding natural language sentences would be ungrammatical. For reasoning along these lines, see NF's speculative discussion of why negation and downward-monotone quantification over questions are unavailable.

\section{Exhaustive questions versus complete answers}

If an assertion $\phi$ entails a question $\psi$, then we say that $\phi$ is a complete answer to $\psi$. For example, asserting that it is raining and nobody is quitting $(\square(R \wedge \forall x . \neg Q x))$ 
completely answers the question who is quitting (?x. $Q x)$.

Completeness relates answers to questions; exhaustivity is a separate notion that applies to $w h$-questions only. The encoding of $w h$-questions that we have presented so far-as formulae of the form $\forall \vec{x}$. ? $\phi$-is strongly exhaustive in that it universally quantifies over $\vec{x}$. To know who is quitting in this sense is to know for each person $x$ either that $x$ is quitting or that $x$ is not. By contrast, to know who is quitting in the weakly exhaustive sense is to know, for each person $x$ who is quitting, that $x$ is quitting: $\forall x . Q x \rightarrow \square Q x$, or more generally $\forall \vec{x} . \phi \rightarrow \square \phi$. Finally, a question like where a gas station is, in an appropriately desperate situation, is non-exhaustive and would be represented by existential quantification: $\exists x . \square G x$. Any assertion of a gas station's location qualifies as a complete answer.

Van Rooy (2003) offers to view the difference between exhaustive and nonexhaustive questions not as ambiguity but rather as underspecification. ${ }^{3} \mathrm{He}$ assigns to questions a uniform meaning regardless of whether they are exhaustive or not, namely a family of (potentially overlapping) sets of possible worlds. These sets are determined in accordance with the optimal answers to the asker's decision problem, the one that motivated asking the question in the first place. This approach has the advantage of unifying questions of different exhaustivity levels. However, it does so at the price of introducing additional notions having to do with the speaker's hidden mental state (such as the underlying decision problem, and a notion of the optimal utility of answers). Our knowledge conditions can be thought to encode the utility of an answer as a binary value: either useful (that is, entailing the knowledge condition) or not.

It is technically possible to reformulate van Rooy's approach in our terms by dividing a subset of the domain (consisting of those elements that are relevant according to van Rooy) into a family $\mathcal{S}$ of possibly overlapping subsets. Intuitively, these are the subsets of equal utility for the asker. The meaning of a question then remains universal quantification, but with an added existential quantification over the subsets in $\mathcal{S}$. For instance, Where can I get gas? becomes: $\exists D^{\prime} \in \mathcal{S} . \forall x \in D^{\prime} . \square G x$. The exhaustive case is when $\mathcal{S}$ consists of a single set: the domain. The nonexhaustive case is when $\mathcal{S}$ is a full partition of the domain into singletons. Presumably, $\mathcal{S}$ can be determined by the same methods as van Rooy determines his division of the set of possible worlds.

Any strongly-exhaustive question $\forall \vec{x}$. ? $\phi$ can be recast as the weakly-exhaustive question

$$
\forall \vec{x} . \forall y \cdot(y=\phi) \rightarrow \square(y=\phi)
$$

(in which the variable $y$ ranges over truth values), because both questions are equivalent to

$$
\forall \vec{x} .(\phi \rightarrow \square \phi) \wedge(\neg \phi \rightarrow \square \neg \phi) .
$$

We can characterize the difference between strongly-, weakly-, and nonexhaustive questions semantically, as follows. Exhaustive questions are additive: if $q$ is an exhaustive question, then $q\left(W_{1}\right)(w) \wedge q\left(W_{2}\right)(w)$ implies $q\left(W_{1} \cup W_{2}\right)(w)$, 
and similarly for infinite unions. In words, a disjunction of complete answers is still a complete answer. Non-exhaustive questions are not additive: Where can I get gas? is completely answered by both in Central Square and in Inman Square, but not by in either Central Square or Inman Square; I'm not sure which. Stronglyexhaustive questions are not only additive but also constant across worlds. That is, $q(W)\left(w_{1}\right)=q(W)\left(w_{2}\right)$, if $q$ is strongly exhaustive, and $w_{1}$ and $w_{2}$ are worlds in $W$.

Let $q$ be an additive question denotation. Following Heim's terminology (1994), we define the answerl of $q$ at a world $w$, written $A_{w}^{1}(q)$, to be the union of all world-sets $W$ satisf ying $q(W)(w)$. The additivity of $q$ ensures that the answerl is itself an answer, in that $q\left(A_{w}^{1}(q)\right)(w)$ is true. Because $q$ is monotonic, $q(W)(w)$ is true just in case $W$ is a subset of $A_{w}^{1}(q)$. Thus $q$ is determined by $A_{w}^{1}(q)$ at each world $w$, and we are tempted to simplify the semantic type of exhaustive questions from $\langle\langle s, t\rangle,\langle s, t\rangle\rangle$ to $\langle s,\langle s, t\rangle\rangle$ : given a world $w$, return the answer1 $A_{w}^{1}(q)$ at $w$. However, this simplification only works for exhaustive questions. Beck and Rullmann (1999; Section 7.1) hence argue that a question must sometimes denote a set of answer propositions, rather than always denoting its answerl proposition. By contrast, our question denotations handle strongly-, weakly-, and non-exhaustive questions all at the same type, namely $\langle\langle s, t\rangle,\langle s, t\rangle\rangle$.

\section{Plurality of questions}

As explained above, we interpret a wh-question by universally quantifying over individual questions. This strategy has a strong connection to Beck and Sharvit's work (2002; Sharvit and Beck 2001) on families of subquestions for explaining quantificational variability effects. On Beck and Sharvit's analysis, sentences like

Alice mostly knows who is quitting.

With few exceptions, Alice knows who is quitting.

quantify over a contextually salient family of subquestions of the question who is quitting. A family of subquestions is simply a set of questions, satisf ying some conditions detailed below. For example, the family $S$ of subquestions might be

\{ is Alice quitting, is Bob quitting, is Carol quitting, ... \},

and the sentences (17) and (18) mean

(20) For most questions $s$ in $S$, Alice knows $s$.

(21) For all but few questions $s$ in $S$, Alice knows $s$.

Beck and Sharvit argue that these sentences quantify over not persons or propositions but questions. Leaving these arguments aside, we show here how our modal perspective expresses their proposal, so as to generalize it to weakly- and non-exhaustive questions as introduced in Section 7. 
It is tempting to view the $w h$-question formula $? \vec{x} . \phi$ as explicitly encoding the set of questions $\left\{? \phi[\vec{d} / \vec{x}] \mid \vec{d} \in D^{n}\right\}$, where $D$ is the domain and $\vec{x}$ consists of $n$ variables. Indeed, this set is a family of subquestions, used in the simplest cases of quantificational variability, such as those above. But as Beck and Sharvit show, this set sometimes differs from the contextually salient family of subquestions. To allow for these cases, Beck and Sharvit propose that any set $S$ of questions can be used as the family of subquestions of a question $q$, as long as the following three criteria are satisfied.

1. Answering every question in $S$ would answer $q$ as well.

2. Every question $s$ in $S$ is a subquestion of $q$. Formally, if $s$ and $q$ both denote partitions of the set of possible worlds into classes, then there is some class of $s$ that is disjoint from some class of $q$. In other words, there are two possible worlds $w_{s}$ and $w_{q}$ such that the partition according to $s$ containing $w_{s}$ is disjoint from the partition according to $q$ containing $w_{q} \cdot{ }^{4}$ Note that, despite the terminology, being a subquestion is a symmetric relation.

3. No proper subset of $S$ satisfies these two criteria.

The second criterion above mentions partitions: Beck and Sharvit only consider strongly exhaustive questions, which they follow GS in treating as partitions of the set of possible worlds. However, the family of subquestions approach is applicable not only to strongly-exhaustive questions but also to weakly- and even (under certain conditions) non-exhaustive questions. Since partitions cannot be used to model weakly- and non-exhaustive questions, we must reformulate the second criterion using only knowledge conditions. Instead of referring to the partitions containing $w_{s}$ and $w_{q}$, we can look at an answer to $s$ in $w_{s}$ and an answer to $q$ in $w_{q}$. These answers must be disjoint, that is, mutually exclusive. This yields the following formulation.

1. The conjunction of all questions in $S$ entails $q$.

2. Every question $s$ in $S$ is a subquestion of $q$, in the sense that there exist two possible worlds $w_{s}$ and $w_{q}$, such that any two propositions $W_{s}$ and $W_{q}$ are mutually exclusive whenever they answer $s$ and $q$ at $w_{s}$ and $w_{q}$, respectively. In other words, for $s$ and $q$ to be subquestions of each other is for there to be worlds $w_{s}$ and $w_{q}$, such that any two world-sets $W_{s}$ and $W_{q}$ are disjoint whenever $s\left(W_{s}\right)\left(w_{s}\right)$ and $q\left(W_{q}\right)\left(w_{q}\right)$ are both true. ${ }^{5}$

3. No proper subset of $S$ satisfies these two criteria.

This reformulation has the virtue of being applicable even when the partition semantics of questions is not.

Despite this reformulation, non-exhaustive questions cannot ordinarily be interpreted as a family of subquestions, as reflected by their distinct (existential) representation. For example, if Alice knows the exact location of even a single gas station, then Alice knows exactly where to get gas. If Alice runs out of gas while 
driving, and she asks Bob whether he knows where to get gas, it would be odd for Bob to answer with few exceptions, I do, even if Bob knows the exact locations of all but a few gas stations in the area. This oddity is because no discrete family of subquestions is contextually salient. Bob could say I mostly do, not to mean that he knows the locations of most gas stations nearby, but to mean that he has somewhat vague recollections of how to navigate to a certain gas station nearby. The relevant family of subquestions here is how to navigate at each step.

\section{Multi-party conversations}

Because the partition theory of questions embeds into our semantics, we can recast Groenendijk's game of interrogation (1999) in our terms. In Groenendijk's original game, an interrogator and a witness take turns asking questions and asserting answers. The interrogator must not ask superfluous questions; for example, having asked Who is quitting?, the interrogator must not ask Who is not quitting? because of the entailment (8) above. The witness must not make irrelevant assertions; for example, having been asked Who is quitting and moving away?, the witness must not assert Alice is not quitting because of the non-entailment (10) above. (The witness must also avoid assertions that are redundant, meaning entailed by the common ground, or incredible, meaning inconsistent with the common ground.) From the modal perspective, we can extend this game from one interrogator and one witness to multiple, overlapping groups of participants.

Groenendijk keeps track of the knowledge and issues in the current common ground in a context $C$, which is a partial equivalence relation over possible worlds, or equivalently, a partition of a subset of the set of possible worlds. A world appears in the context just in case it is not yet ruled out by the knowledge state in the common ground; two worlds are related by the context just in case it is not yet under discussion which one is real. In Groenendijk's game, the only knowledge state and knowledge conditions relevant to felicity in the conversation is the common ground between the interrogator and the witness. We can generalize this to knowledge state and knowledge conditions among overlapping groups by keeping track of one context per group. That is, for every group of participants $G$, we keep track of a context $C_{G}$ for that group, still a partial equivalence relation over worlds. Entailment among contexts respects containment among groups; that is, if $G^{\prime} \subseteq G$, then $C_{G^{\prime}} \subseteq C_{G}$ throughout our generalized game.

Groenendijk's interrogator updates the context by removing equivalences; his witness updates the contexts by removing worlds. For a question $q$ (denoting a partition of worlds) to be non-redundant is for the context $C$ to not entail $q$. For an assertion $\phi$ (denoting a set of worlds) to be relevant is for the context $C$ to entail ? $\phi$, where ? $\phi$ is the binary partition of worlds formed by $\phi$ and its complement $\neg \phi$. In our generalization, the group $G$ of participants in the room can change from move to move. A question still removes equivalences, and an answer still removes worlds, but from every context $C_{G^{\prime}}$ for subgroups $G^{\prime}$ of $G$. (It is easy to check that this update procedure preserves the invariant that entailment among contexts 
respects containment among groups.) Each question $q$ must not be redundant: $C_{G}$ better not entail $q$. (Because of the invariant, then, it never hurts to ask a question in a bigger group. For example, the same question may be asked again after a participant enters, but not leaves, the room.) Each answer $\phi$ must be relevant: $C_{G}$ better entail $? \phi$. (Because of the invariant, it never hurts to assert an answer in a smaller group---except the assertion may then be redundant or incredible.)

The generalization just described of Groenendijk's game may seem straightforward at first glance, and to the extent that it is straightforward, the link that this paper makes between question meanings and knowledge conditions is successful. However, we must point out two caveats that call for future work.

First and foremost, neither Groenendijk's nor our game deals with questions that are not strongly exhaustive. The problem lies with the licensing condition for answers: according to Groenendijk, an answer $\phi$ is relevant to assert just in case the context entails ? $\phi$. But if a question $\psi$ is under discussion that is weakly- or non-exhaustive, then even a complete answer $\phi$ to $\psi$ (that is, so that $\square \phi \vDash \psi$ ) is considered irrelevant (that is, $\psi \forall ? \phi$ )! This wrongly prevents any answer to a question like Where can I get gas?. Hence this relevance criterion must be revised for nonstrongly-exhaustive questions. Invoking the notion of subquestions, perhaps one could err on the side of permissiveness and allow any answer $\phi$ to be asserted as long as $\square \phi$ completely answers any subquestion in any family of subquestions of $\psi$.

Second, as we consider more complex games of interrogation, it becomes less clear that our formal criteria for redundancy and relevance really correspond to intuitive notions. In what sense do participants in these games know what issues are under discussion, and work towards the goal of resolving these issues? Not in any sense so far formally related to logics for epistemic actions. Grounding games of interrogation like ours, in logics for epistemic actions such as Baltag et al.'s (1999), would help model subtleties such as the following. Suppose that Alice asks Bob and Carol whether it is raining, then Bob leaves the room. Is it still relevant for Carol to tell Alice that it is raining? This assertion does not further common knowledge among Alice, Bob, and Carol, yet our generalized game above allows it.

\section{Endnotes}

1. For completeness, here is the list of axioms:

Necessitation If $\phi$ is provable with no assumption, then so is $\square \phi$.

$\mathbf{K} \square(\phi \rightarrow \psi) \rightarrow \square \phi \rightarrow \square \psi$.

The $\mathbf{T}$ axiom $\square \phi \rightarrow \phi$.

Barcan both ways $(\square \forall x . \phi) \leftrightarrow(\forall x . \square \phi)$.

The 4 axiom $\square \phi \rightarrow \square \square \phi$;

The B axiom $\phi \rightarrow \square \neg \square \neg \phi$.

2. A bilattice is a set that is simultaneously ordered by two partial orders.

3. We thank one of the anonymous reviewers for directing our attention to this point. 
4. If, to the contrary, every partition of $s$ intersects every partition of $q$, then $s$ and $q$ are unrelated questions.

5. Recall that, if $q$ is a question, $W$ is a set of worlds, and $w$ is a world, then $q(W)(w)$ holds if knowing that the actual world is in $W$ entails knowing the complete answer to $q$ at $w$.

* We thank Nissim Francez, Stuart Shieber, the anonymous reviewers and the participants of the SALT 14 conference for their suggestions and comments on an earlier version of this paper.

\section{References}

Åqvist, Lennart. 1965. A new approach to the logical theory of interrogatives. Uppsala: Almqvist \& Wiksell.

Baltag, Alexandru, Lawrence S. Moss, and Slawomir Solecki. 1999. The logic of public announcements, common knowledge and private suspicions. Tech. Rep. 534, Computer Science Department, Indiana University.

Beck, Sigrid, and Hotze Rullmann. 1999. A flexible approach to exhaustivity in questions. Natural Language Semantics 7(3):249-298.

Beck, Sigrid, and Yael Sharvit. 2002. Pluralities of questions. Journal of Semantics 19(2):105-157.

ten Cate, Balder, and Chung-chieh Shan. 2002. Question answering: From partitions to Prolog. In Proceedings of TABLEAUX 2002: Automated reasoning with analytic tableaux and related methods, ed. Uwe Egly and Christian G. Fermüller, 251-265. Lecture Notes in Computer Science 2381, Berlin: Springer-Verlag.

Cerrito, Serenella, and Marta Cialdea Mayer. 2001. Free-variable tableaux for constant-domain quantified modal logics with rigid and non-rigid designation. In Proceedings of IJCAR 2001: 1st international joint conference on automated reasoning, ed. Rajeev Goré, Alexander Leitsch, and Tobias Nipkow, 137-151. Lecture Notes in Computer Science 2083, Berlin: SpringerVerlag.

Groenendijk, Jeroen. 1999. The logic of interrogation: Classical version. In SALT IX: Semantics and linguistic theory, ed. Tanya Matthews and Devon Strolovitch, 109-126. Ithaca: Cornell University Press.

Groenendijk, Jeroen, and Martin Stokhof. 1984. Studies on the semantics of questions and the pragmatics of answers. Ph.D. thesis, Universiteit van Amsterdam.

- 1996. Questions. In Handbook of logic and language, ed. Johan van Benthem and Alice ter Meulen, 1055-1124. Amsterdam: Elsevier Science.

Hamblin, Charles Leonard. 1973. Questions in Montague English. Foundations of Language 10:41-53.

Heim, Irene. 1994. Interrogative semantics and Karttunen's semantics for know. In Proceedings of the Israeli association for theoretical linguistics.

Hintikka, Jaakko. 1976. The semantics of questions and the questions of semantics. 
Acta Philosophica Fennica 28(4).

Karttunen, Lauri. 1977. Syntax and semantics of questions. Linguistics and Philosophy 1(1):3-44.

Nelken, Rani, and Nissim Francez. 2000. A calculus of interrogatives based on their algebraic semantics. In Proceedings of TWLT16/AMILP2000: Algebraic methods in language processing, ed. Dirk Heylen, Anton Nijholt, and Giuseppe Scollo, 143-160.

-2002. Bilattices and the semantics of natural language questions. Linguistics and Philosophy 25(1):37-64.

van Rooy, Robert. 2003. Questioning to resolve decision problems. Linguistics and Philosophy 26(6):727-763.

Sharvit, Yael, and Sigrid Beck. 2001. Subquestions and quantificational variability effects. In Proceedings of the 20th West Coast Conference on Formal Linguistics, ed. Karine Megerdoomian and Leora A. Bar-el, 510-523. Somerville, MA: Cascadilla.

Szabolcsi, Anna. 1997. Quantifiers in pair-list readings. In Ways of scope taking, ed. Anna Szabolcsi, chap. 9, 311-347. Dordrecht: Kluwer. 